\title{
铝掺杂及铇酸锂表面包覆双效提升富锂锰基正极材料的循环稳定性
}

\author{
任旭强李东林* 赵珍珍 陈光琦 赵坤 孔祥泽 李童心 \\ (长安大学材料科学与工程学院 西安 710061)
}

\begin{abstract}
摘要 采用溶胶一凝胶法合成 $\mathrm{Al}$ 掺杂富锂锰基 $\mathrm{Li}_{1.2} \mathrm{Mn}_{0.54-x} \mathrm{Al}_{x} \mathrm{Ni}_{0.13} \mathrm{Co}_{0.13} \mathrm{O}_{2}(x=0 、 0.03)$ 锂离子电池正极材料, 之后采用 一步液相法制备 $\mathrm{Li}_{2} \mathrm{WO}_{4}$ 包覆层, 系统地研究了 $\mathrm{Al}$ 掺杂和 $\mathrm{Li}_{2} \mathrm{WO}_{4}$ 包覆双效改性对富锂锰基正极材料电化学性能的影 响. 结果表明, $\mathrm{Al}$ 掺杂后明显提升富锂锰基正极材料的循环稳定性, 包覆层 $\mathrm{Li}_{2} \mathrm{WO}_{4}$ 明显改善其倍率性能和放电平台电 压衰减问题. $\mathrm{Li}_{2} \mathrm{WO}_{4}$ 包覆量为 $5 \% \mathrm{Li}_{1.2} \mathrm{Mn}_{0.51} \mathrm{Al}_{0.03} \mathrm{Ni}_{0.13} \mathrm{Co}_{0.13} \mathrm{O}_{2}$ 正极材料在 $2.0 \sim 4.8 \mathrm{~V}$ 充放电电压区间及 $1000 \mathrm{~mA} \cdot \mathrm{g}^{-1}$ 电流密度下比容量仍高达 $110 \mathrm{mAh} \cdot \mathrm{g}^{-1}$ 左右，同时在 $100 \mathrm{~mA} \cdot \mathrm{g}^{-1}$ 的电流密度下循环 300 次容量保持率为 $78 \%$, 而且循 环过程中放电平台电压衰减也明显减缓. 该工作为解决锂离子电池富锂锰基正极材料循环稳定性和平台电压衰减提供 了新的思路.
\end{abstract}

关键词 锂离子电池; 溶胶-凝胶法; 富锂锰基正极材料; $\mathrm{Li}_{2} \mathrm{WO}_{4} ; \mathrm{Al}$ 掺杂

\section{Dual Effect of Aluminum Doping and Lithium Tungstate Coating on the Surface Improves the Cycling Stability of Lithium-rich Manganese-based Cathode Materials}

\author{
Ren, Xuqiang Li, Donglin* Zhao, Zhenzhen Chen, Guangqi Zhao, Kun \\ Kong, Xiangze Li, Tongxin \\ (School of Materials Science and Engineering, Chang'an University, Xi'an 710061, China)
}

\begin{abstract}
The layered lithium-rich manganese-based cathode material has been widely concerned because of it's advantages such as a specific discharge capacity greater than $250 \mathrm{mAh} \cdot \mathrm{g}^{-1}$ and a high discharge platform, and is expected to become the next-generation lithium ion battery cathode material. However, lithium-rich manganese-based cathode materials have poor cycle stability, low coulombic efficiency for the first charge and discharge, and low rate performance. The most important thing is that the problem of faster voltage decays on the discharge platform has not been effectively solved, The current it is mainly to improve the performance by element doping modification and surface coating, so it is very important to find suitable doping elements and coating materials. The common coating material is mainly to prevent direct contact between the electrolyte and the positive electrode material to improve the cycle stability, and it is difficult to slow down the attenuation of the discharge voltage platform. Al-doping lithium-rich manganese-based $\mathrm{Li}_{1.2} \mathrm{Mn}_{0.54-x} \mathrm{Al}_{x} \mathrm{Ni}_{0.13} \mathrm{Co}_{0.13} \mathrm{O}_{2}(x=0,0.03)$ cathode material was prepared by sol-gel method and $\mathrm{Li}_{2} \mathrm{WO}_{4}$ surface coating by one-step liquid phase method. The required materials were confirmed by X-ray diffractometer (XRD), energy disperse spectroscopy (EDS) and scanning electron microscope (SEM) tests, and then the effects of Al-doping and $\mathrm{Li}_{2} \mathrm{WO}_{4}$ coated double-effect modification on the electrochemical performance of lithium-rich manganese-based cathode materials were studied by electrochemical test system. The results show that Al doping significantly improves the cycling stability of lithium-rich manganese-based cathode materials, and the coating $\mathrm{Li}_{2} \mathrm{WO}_{4}$ significantly improves its rate performance and discharge platform voltage attenuation. In $5 \% \mathrm{Li}_{2} \mathrm{WO}_{4}$ coated $\mathrm{Li}_{1.2} \mathrm{Mn}_{0.51} \mathrm{Al}_{0.03} \mathrm{Ni}_{0.13} \mathrm{Co}_{0.13} \mathrm{O}_{2}$ cathode material in charge and discharge voltage $2.0 \sim 4.8 \mathrm{~V}$, and under the current density $1000 \mathrm{~mA} \cdot \mathrm{g}^{-1}$, the specific capacity is still as high as about $110 \mathrm{mAh} \cdot \mathrm{g}^{-1}$. At the same time, the specific capacity retention rate was $78 \%$ after 300 cycles at the current density of $100 \mathrm{~mA} \cdot \mathrm{g}^{-1}$, and the voltage decay of the discharge platform significantly slowed down during the cycle.

Keywords lithium ion battery; sol-gel; lithium-rich manganese-based cathode material; $\mathrm{Li}_{2} \mathrm{WO}_{4}$; $\mathrm{Al}$-doping
\end{abstract}

\section{1 引言}

高能量密度的锂离子电池一直是人们在移动终端、 电动汽车等方面所追求的目标, 在现有的电池技术中, 已经商用石墨负极材料具有超过 $350 \mathrm{mAh} \cdot \mathrm{g}^{-1}$ 的放电容
量, 而正极材料仅有 $140 \sim 170 \mathrm{mAh} \cdot \mathrm{g}^{-1}$, 因此限制锂离 子电池能量密度的因素主要是正极材料 ${ }^{[1]}$. 目前所商用 的正极材料主要有: $\mathrm{LiFePO}_{4} 、 \mathrm{LiCoO}_{2}$ 等, $\mathrm{LiFePO}_{4}$ 安全 性能良好、循环稳定，但其 $170 \mathrm{mAh} \cdot \mathrm{g}^{-1}$ 的理论容量很 难满足长续航电动汽车的续航要求; $\mathrm{LiCoO}_{2}$ 性能稳定,

\footnotetext{
* E-mail: dlli@chd.edu.cn; Tel.: 029-82337340

Received July 16, 2020; published October 9, 2020.

Project supported by the National Natural Science Foundation of China (Nos. 21473014, 21073021).

项目受国家自然科学基金面上项目(Nos. 21473014, 21073021)资助.
} 
然而有价格昂贵、钴资源短缺、污染环境等缺点 ${ }^{[2-5]}$. 针 对这些问题并更好地满足电动汽车的续航里程要求, 层 状富锂锰基正极材料 $x \mathrm{Li}_{2} \mathrm{MnO}_{3} \cdot(1-x) \mathrm{LiMO}_{2}(0<x<1$, $\mathrm{M}=\mathrm{Mn} 、 \mathrm{Co} 、 \mathrm{Ni} .$.$) 可以被认为是 \mathrm{LiMO}_{2}(\mathrm{M}=\mathrm{Mn} 、 \mathrm{Co}$ 、 Ni) 和 $\mathrm{Li}_{2} \mathrm{MnO}_{3}$ 组成的固溶体或者复合氧化物, 它由于 具有大于 $250 \mathrm{mAh} \cdot \mathrm{g}^{-1}$ 的放电比容量和放电平台高等优 势而得到广泛关注, 有望成为下一代锂离子电池正极材 料 ${ }^{[6]}$. 但是, 富锂锰基正极材料循环稳定性差、首次充放 电库仑效率低、倍率性能差, 最重要的是其放电平台电 压衰减较快的问题一直无法得到有效解决，从而限制了 它的使用 ${ }^{[7]}$.

富锂锰基正极材料性能差是因为充放电过程中表 面发生相变以及结构不稳定 ${ }^{[8]}$, 目前对于其的研究主要 集中在元素掺杂改性和表面包覆等. 元素的掺杂改性可 以提高正极材料的结构稳定性、减小 $\mathrm{Li}^{+}$迁移的阻碍以 及抑制不利相变等方式, 来提高材料的电化学性能 ${ }^{[9-10]}$, 常见的掺杂离子主要是阳离子, 例如 $\mathrm{Na}^{+[11]} 、 \mathrm{~K}^{+[12]}$ 、 $\mathrm{Mg}^{2+[13]} 、 \mathrm{Ru}^{4+[14]} 、 \mathrm{Ti}^{4+[15]}$ 及 $\mathrm{Mo}^{6+[16-17]}$ 等，同时也有像 $\mathrm{F}^{-[18]}$ 和 $\mathrm{S}^{2-[19]}$ 的阴离子掺杂, 还可以用 $\mathrm{Mg}^{2+}$ 和 $\mathrm{F}^{-}$进行 阴阳离子共掺杂 ${ }^{[20]}$. 值得一提的是, 表面包覆可以阻止 富锂锰基材料与电解液直接接触破坏材料的结构, 减少 了副反应的发生进而提高了材料的电化学性能 ${ }^{[21]}$, 常 见的表面包覆物有 $\mathrm{ZrO}_{2}{ }^{[22]} 、 \mathrm{TiO}_{2}{ }^{[23]} 、 \mathrm{Al}_{2} \mathrm{O}_{3}{ }^{[24]}$ 等氧化物, 也有 $\mathrm{AlF}_{3}{ }^{[25]} 、 \mathrm{AlPO}_{4}{ }^{[26]} 、 \mathrm{LaF}_{2}{ }^{[27]}$ 以及 $\mathrm{C}^{[28]}$ 等材料, 但是 这些包覆材料很难对平台衰减有很大改善, 因此需要进 一步研究并寻找其它包覆层材料. 掺杂和包覆改性材料 与合成方法有关, 富锂锰基正极材料可采用高温固 相 $^{[29]}$ 、共沉淀 ${ }^{[30]}$ 、溶胶-凝胶 ${ }^{[31]}$ 、水热 ${ }^{[32]}$ 等方法来合成, 合成条件对富锂锰基材料结构和电化学性能也有重要 的影响, 溶胶-凝胶可以实现原子级别的均匀混合, 而 且有操作简单、烧结温度低、产物的颗粒尺寸小且粒度 均一、比表面积较大等优点. 鉴于此, Song 等 ${ }^{[31]}$ 通过溶 胶-凝胶法合成的 $\mathrm{Li}_{1.2} \mathrm{Mn}_{0.54} \mathrm{Ni}_{0.13} \mathrm{Co}_{0.13} \mathrm{O}_{2}$ 在 $400 \mathrm{~mA} \cdot \mathrm{g}^{-1}$ 电流密度下循环 100 次后仍有 $133.7 \mathrm{mAh} \cdot \mathrm{g}^{-1}$ 的比容量, 容量保持率达 $84.14 \%$.

$\mathrm{Li}$ 等 ${ }^{[33-34]}$ 采用溶胶-凝胶法将 $\mathrm{Al}$ 掺杂到富锂锰基正 极材料中有效地改善了其循环性能, 经研究表明 $\mathrm{Al}$ 掺 杂可以稳定富锂锰基正极材料的晶体结构改善循环性 能, 而且可以促进电荷转移和减少阻抗提高富锂锰基正 极材料的倍率性能. 同时, 现有研究表明 $\mathrm{Li}_{2} \mathrm{WO}_{4}$ 具有 良好的 $\mathrm{Li}^{+}$导电性 ${ }^{[35-36]}$, Hayashi 等 ${ }^{[37-39]}$ 将非晶态的 $\mathrm{Li}_{2} \mathrm{WO}_{4}$ 沉积在 $\mathrm{LiCoO}_{2}$ 正极材料表面可以降低锂离子电 池的界面电阻, 而且可以防止电解液直接和正极材料接 触减少副反应进而改善其电化学性能, 但是目前对于 $\mathrm{Li}_{2} \mathrm{WO}_{4}$ 表面改性 $\mathrm{Li}_{1.2} \mathrm{Mn}_{0.54} \mathrm{Ni}_{0.13} \mathrm{Co}_{0.13} \mathrm{O}_{2}$ 鲜有报道. 因 此, 本工作用 $\mathrm{Al}$ 掺杂和 $\mathrm{Li}_{2} \mathrm{WO}_{4}$ 表面包覆同时改性 $\mathrm{Li}_{1.2} \mathrm{Mn}_{0.54} \mathrm{Ni}_{0.13} \mathrm{Co}_{0.13} \mathrm{O}_{2}$ 正极材料. 结果显示, 包覆 $5 \%$ 的 $\mathrm{Li}_{2} \mathrm{WO}_{4}$ 的 $\mathrm{Li}_{1.2} \mathrm{Mn}_{0.49} \mathrm{Al}_{0.03} \mathrm{Ni}_{0.13} \mathrm{Co}_{0.13} \mathrm{O}_{2}$ 具有良好倍率性
能, 在 $100 \mathrm{~mA} \cdot \mathrm{g}^{-1}$ 的电流密度下循环 300 次容量保持率 能达到 $78 \%$, 比容量仍有 $150 \mathrm{mAh} \cdot \mathrm{g}^{-1}$, 纯的富锂锰基 正极材料的容量保持率仅有 $60.4 \%$, 更重要的是, 该包 覆层对倍率性能以及放电平台的衰减也有明显的改善. 这为今后富锂锰基正极材料的研究提供了一个思路.

\section{2 结果与讨论}

\section{$2.1 X$ 射线衍射分析}

图 1 是三种材料(为了方便下文将 $\mathrm{Li}_{1.2} \mathrm{Mn}_{0.54} \mathrm{Ni}_{0.13}$ $\mathrm{Co}_{0.13} \mathrm{O}_{2} 、 \mathrm{Li}_{1.2} \mathrm{Mn}_{0.51} \mathrm{Al}_{0.03} \mathrm{Ni}_{0.13} \mathrm{Co}_{0.13} \mathrm{O}_{2}$ 和 $5 \% \mathrm{Li}_{2} \mathrm{WO}_{4} @$ $\mathrm{Li}_{1.2} \mathrm{Mn}_{0.51} \mathrm{Al}_{0.03} \mathrm{Ni}_{0.13} \mathrm{Co}_{0.13} \mathrm{O}_{2}$ 分别记为 LMNC-1、 $\mathrm{LMNC}-2$ 和 LMNC-3) 的 XRD 衍射图谱以及具有 $R \overline{3} \mathrm{~m}$ 空 间结构群的 $\mathrm{Li}_{2} \mathrm{WO}_{4}$ 的标准卡片 PDF\#22-0693. 由图可 知, 三种材料均有富锂锰基材料的所有特征峰, 属于三 方晶系, $R \overline{3} m$ 空间群 ${ }^{[17-18]}$, 其中在 $2 \theta$ 为 $20^{\circ} \sim 25^{\circ}$ 处, 出 现了一些强度较弱的衍射峰, 这些是由于 $\mathrm{Li}$ 和 $\mathrm{Mn}$ 超晶 格排序造成的，确定了 $\mathrm{Li}_{2} \mathrm{MnO}_{3}$ 组分(空间群: $C 2 / m$ ) 存 在, 这一结果与 Xiang 等 ${ }^{[11-15]}$ 之前的研究一致, 可以看 到在 $(006) /(102)$ 和 $(018) /(110)$ 晶面的衍射峰有较为明显 的分裂, 这是由于 $\mathrm{LiMO}_{2}$ 中 $\alpha-\mathrm{NaFeO}_{2}$ 层状结构的氧亚 晶格造成, 可以表明经过掺杂和包覆改性的材料依然具 有较好的 $\alpha-\mathrm{NaFeO}_{2}$ 层状结构, 没有改变富锂锰基材料 原有的具备电化学活性的相结构 ${ }^{[40-43]}$. 对比 $\mathrm{Li}_{2} \mathrm{WO}_{4}$ 的 标准卡片发现, LMNC-3 在(111)、(210)、(211)、(220)、 (300)、(310)、(410)、(331)、(420)、(521)和(440)晶面具 有非常明显的衍射峰, 证明 $\mathrm{Li}_{2} \mathrm{WO}_{4}$ 成功包在富锂材料 上. 由于 $\mathrm{Al}^{3+}$ 和 $\mathrm{Mn}^{4+}$ 的半径相同(均为 $0.053 \mathrm{~nm}$ ), 所以 为证明 $\mathrm{Al}$ 掺杂在锰位置上, 对 LMNC-1 与 LMNC-2 的 $\mathrm{XRD}$ 进行了结构精修(图 2). 图中 $R_{\mathrm{wp}}$ 为可靠性因子, $\mathrm{CHI}^{2}$ 为拟合优度. 精修结果显示 LMNC-1 的 $R_{\mathrm{wp}}=$ $3.92 \%, \mathrm{CHI}^{2}=1.343, \mathrm{LMNC}-2$ 的 $R_{\mathrm{wp}}=4.41 \%, \mathrm{CHI}^{2}=$ 1.706. 这符合精修结果可靠判断标准: $R_{\mathrm{wp}}<10 \%$, $\mathrm{CHI}^{2}<2^{[44]}$. 可以说明 $\mathrm{Al}$ 确实掺杂在锰位上.

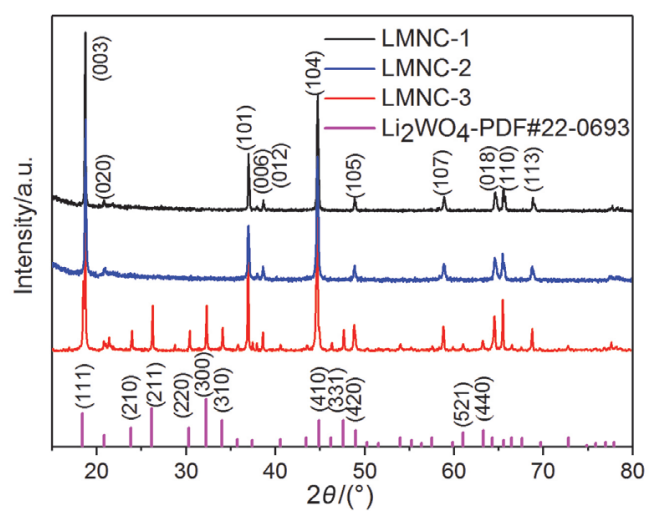

图 1 LMNC-1, LMNC-2 和 LMNC-3 材料的 XRD 图

Figure 1 X-ray diffraction patterns for the LMNC-1, LMNC-2 and LMNC-3 samples 

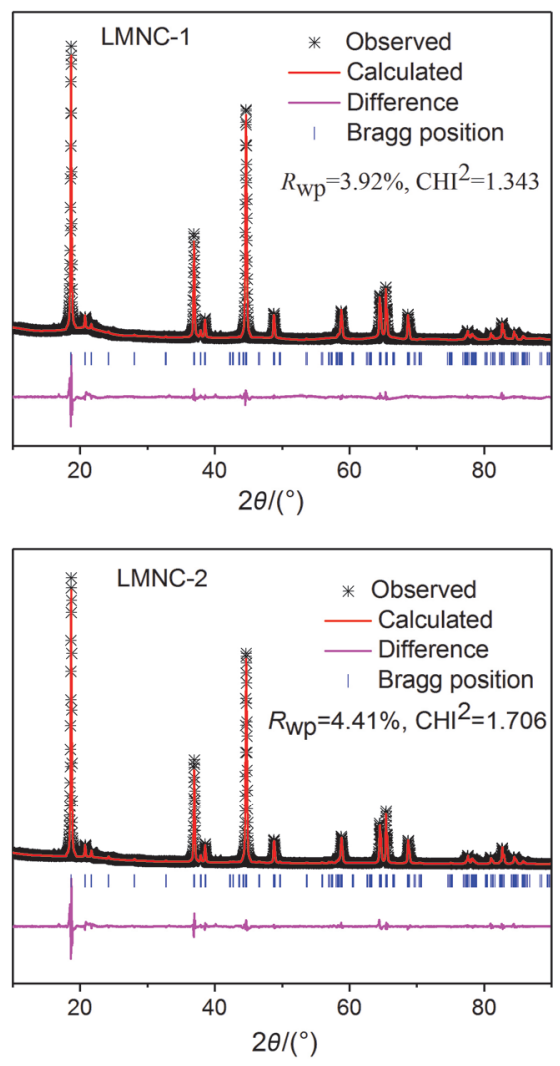

图 2 XRD 精修结果

Figure 2 Rietveld refinement results for XRD data

\section{2 电极材料形貌分析}

图 3 是三种材料的扫描电镜图(SEM)图. 由 $\mathrm{b} 、 \mathrm{~d} 、 \mathrm{f}$ 中可以看出, 未包覆的 LMNC 颗粒均匀, 都有明显团聚 现象, 三种材料出现小颗粒团聚在一块形成二次颗粒, 这样有利于电解液润湿, 使 $\mathrm{Li}^{+}$更加容易扩散进材料中. 从 a、c、e 图中发现, 未包覆 $\mathrm{Li}_{2} \mathrm{WO}_{4}$ 的 LMNC-1 和 LMNC-2 材料棱角分明, 颗粒尺寸在 $150 \sim 300 \mathrm{~nm}$, 然 而包覆 $\mathrm{Li}_{2} \mathrm{WO}_{4}$ 后材料的尺寸有所增大，达到了 $500 \mathrm{~nm}$ 左右, 而且材料的表面棱角不明显, 比较光滑, 也可以 看到有 $10 \mathrm{~nm}$ 左右的小颗粒附着, 这是由于采用液相包 覆的方法, 不能将 $\mathrm{Li}_{2} \mathrm{WO}_{4}$ 均匀包覆在所有富锂锰基正 极颗粒表面从而形成 $\mathrm{Li}_{2} \mathrm{WO}_{4}$ 颗粒, 也表明 $\mathrm{Li}_{2} \mathrm{WO}_{4}$ 在富 锂锰基正极材料表面.

图 4a 和图 $4 \mathrm{~b}$ 分别是 LMNC-2 和 LMNC-3 材料的 EDS 能谱分析结果, 选取样品中一区域扫描分析可以发 现 $\mathrm{Mn} 、 \mathrm{Ni} 、 \mathrm{Co} 、 \mathrm{Al}$ 在材料颗粒中均匀分布, 没有元素 偏聚的现象. 图 $4 \mathrm{~b}$ 中 $\mathrm{W}$ 元素也均匀分布在材料颗粒中, 结合 SEM 图、 EDS 图和 XRD 图可以说明 $\mathrm{Al}$ 元素均匀 地掺杂在 $\mathrm{LMNC}$ 材料中, $\mathrm{Li}_{2} \mathrm{WO}_{4}$ 较好地包覆在掺杂的 材料表面.

\section{3 电化学性能分析}

图 5 中 $\mathrm{a} 、 \mathrm{~b}$ 、和 $\mathrm{c}$ 分别是三种材料在不同电流密度 下的充放电曲线. 在第一圈充放电过程中, 电压从起始
电压到 $4.4 \mathrm{~V}$ 左右是 $\mathrm{LiMO}_{2}$ 中 $\mathrm{Li}^{+}$的脱出，同时过渡金 属离子 $\mathrm{Ni}^{2+}$ 氧化成 $\mathrm{Ni}^{4+} 、 \mathrm{Co}^{3+}$ 氧化成 $\mathrm{Co}^{4+}$, 该过程中 $\mathrm{LiMnO}_{3}$ 中的 $\mathrm{Li}^{+}$也扩散到已经失去 $\mathrm{Li}^{+}$的 $\mathrm{LiMO}_{2}$ 中; 当 电压充到 $4.5 \mathrm{~V}$ 左右时, 材料晶格中发生不可逆的结构 变化, 此时失去 $\mathrm{Li}$ 的 $\mathrm{LiMnO}_{3}$ 被活化成 $\mathrm{MnO}_{2}$, 在放电 过程中 $\mathrm{MnO}_{2}$ 也会提供相应的容量, 这也是富锂锰基典 型的首圈充放电曲线 ${ }^{[41]}$, 三种材料的曲线相似可以说 明经过掺杂和包覆改性的材料没有改变原有的富锂锰 基材料的相结构. 由图可以知道 LMNC-1、LMNC-2 和 LMNC-3 三种材料的首次放电比容量分别是 274.7、 253.0 和 $260.7 \mathrm{mAh} \cdot \mathrm{g}^{-1}$, 对应的首次充放电效率为 $72 \% 、 70 \%$ 和 $73 \%, \mathrm{Al}$ 掺杂后材料的首次充放电效率没

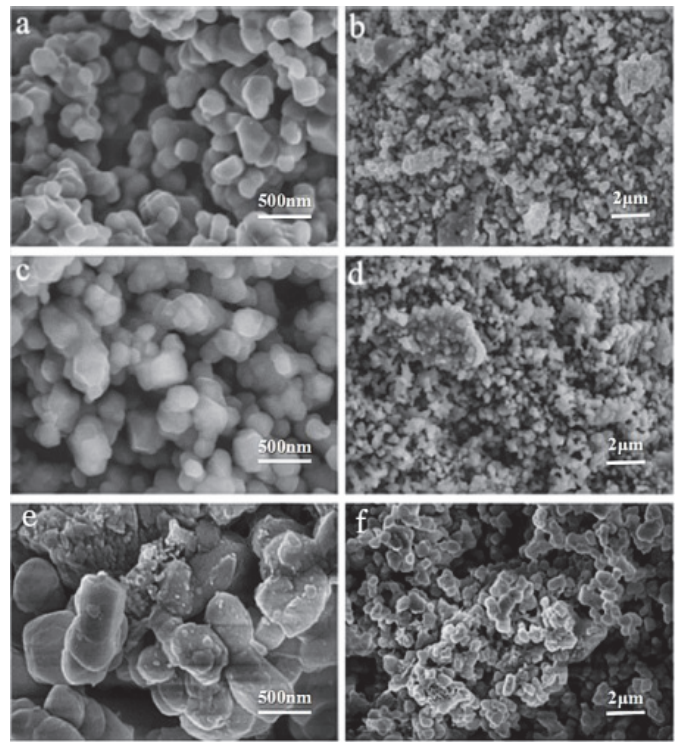

图 3 LMNC-1 (a, b), LMNC-2 (c, d) 和 LMNC-3 (e, f) 材料的 SEM 照片 Figure 3 SEM photographs of LMNC-1 (a, b), LMNC-2 (c, d) and LMNC-3 (e, f) materials

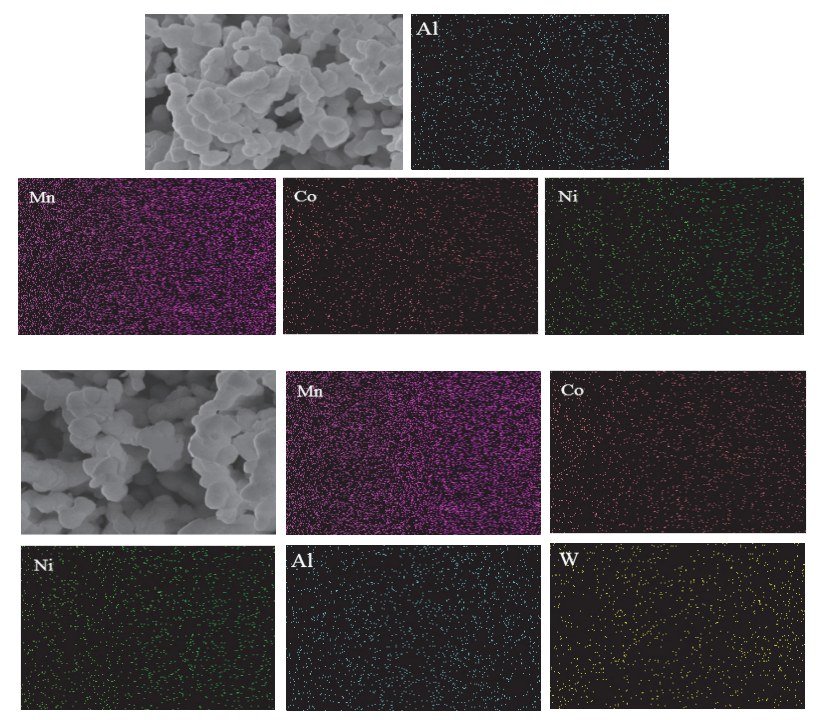

图 4 LMNC-2 和 LMNC-3 材料的 EDS 照片

Figure 4 EDS photo of LMNC-2 and LMNC-3 materials 
有提高, 这是由于 $\mathrm{Al}$ 本身是非电化学活性的; 然而包覆 后材料首次充放电效率略有提高, 这是因为 $\mathrm{Li}_{2} \mathrm{WO}_{4}$ 具 有良好的 $\mathrm{Li}^{+}$导电性, 保证更多的 $\mathrm{Li}^{+}$脱嵌. 结合不同倍 率循环图 5d 可知 $\mathrm{Al}$ 掺杂后会降低放电比容量, 这是由 于 $\mathrm{Al}$ 掺杂代替了 $\mathrm{Mn}$, 减少了氧化还原活性的物质 ${ }^{[45]}$, 而且由于 $\mathrm{Al}^{3+}$ 的半径与 $\mathrm{Co}^{3+}$ 的半径相近, 也会有少量 的 $\mathrm{Al}$ 会代替 $\mathrm{Co}$ 的位置, 这样也会降低材料的放电比容 量; 然而在 $1 \mathrm{C}$ 和 $2 \mathrm{C}$ 倍率下 $\mathrm{Al}$ 掺杂有明显高的比容量, 这是由于 $\mathrm{Al}$ 的掺杂减小的晶胞参数, 稳定材料的结构, 保证了更多锂离子的嵌入 ${ }^{[42]}$.

根据图 $5 \mathrm{~d}$ 可知同时进行 $\mathrm{Al}$ 掺杂与 $\mathrm{Li}_{2} \mathrm{WO}_{4}$ 包覆双 效改性的材料具有更高的比容量, 在 $10 \mathrm{C}$ 下还有近 70 $\mathrm{mAh} \cdot \mathrm{g}^{-1}$ 的放电比容量, 这是由于 $\mathrm{Li}_{2} \mathrm{WO}_{4}$ 可以降低界 面阻抗, 而且其也具有良好的 $\mathrm{Li}^{+}$的导电性, 加快 $\mathrm{Li}^{+}$脱 嵌, 保证更多的 $\mathrm{Li}^{+}$参与电化学反应, 改善了材料的倍 率性能, 这也对应了后文中所提到的 LMNC-3 有最小的
界面阻抗 ${ }^{[37-39]}$ (图 8). 重要的是富锂锰基更需要关注放 电平台衰减问题，图 5 的 e、f 分别是 LMNC-1、LMNC-2 和 LMNC-3 三种材料在 $0.2 \mathrm{C}$ 和 $2 \mathrm{C}$ 下的平台对比图. 从 $\mathrm{e}$ 图中可以发现 LMNC-3 的充电平台最低、放电平台最 高, 这样有利于 $\mathrm{Li}^{+}$在材料中的脱嵌; 从 $\mathrm{f}$ 图中发现在 $2 \mathrm{C}$ 大倍率更加明显，未改性的材料几乎没有放电平台， 比容量也很低, 而 LMNC-3 有明显的放电平台, 比容量 仍有 $150 \mathrm{mAh} \cdot \mathrm{g}^{-1}$, 这是由于 $\mathrm{Al}$ 掺杂与 $\mathrm{Li}_{2} \mathrm{WO}_{4}$ 包覆双 效改性后稳定了材料结构, 这一点后文中的微分电容曲 线图也进一步说明(图 7).

图 $6 \mathrm{a}$ 是 LMNC-1、LMNC-2 和 LMNC-3 三种材料 在 $0.5 \mathrm{C}$ 下长循环性能和不同循环次数下平台衰减对比 图. 从 $\mathrm{a}$ 图中可以看出经过循环 300 圈后三种材料的容 量均在减少，三种材料的容量保持率分别是 $60.0 \%$ 、 $82.0 \% 、 78.0 \%$ ，未改性的容量保持率最低，同时经过掺 杂和包覆的 LMNC-3 容量从 $195 \mathrm{mAh} \cdot \mathrm{g}^{-1}$ 下降到了 151
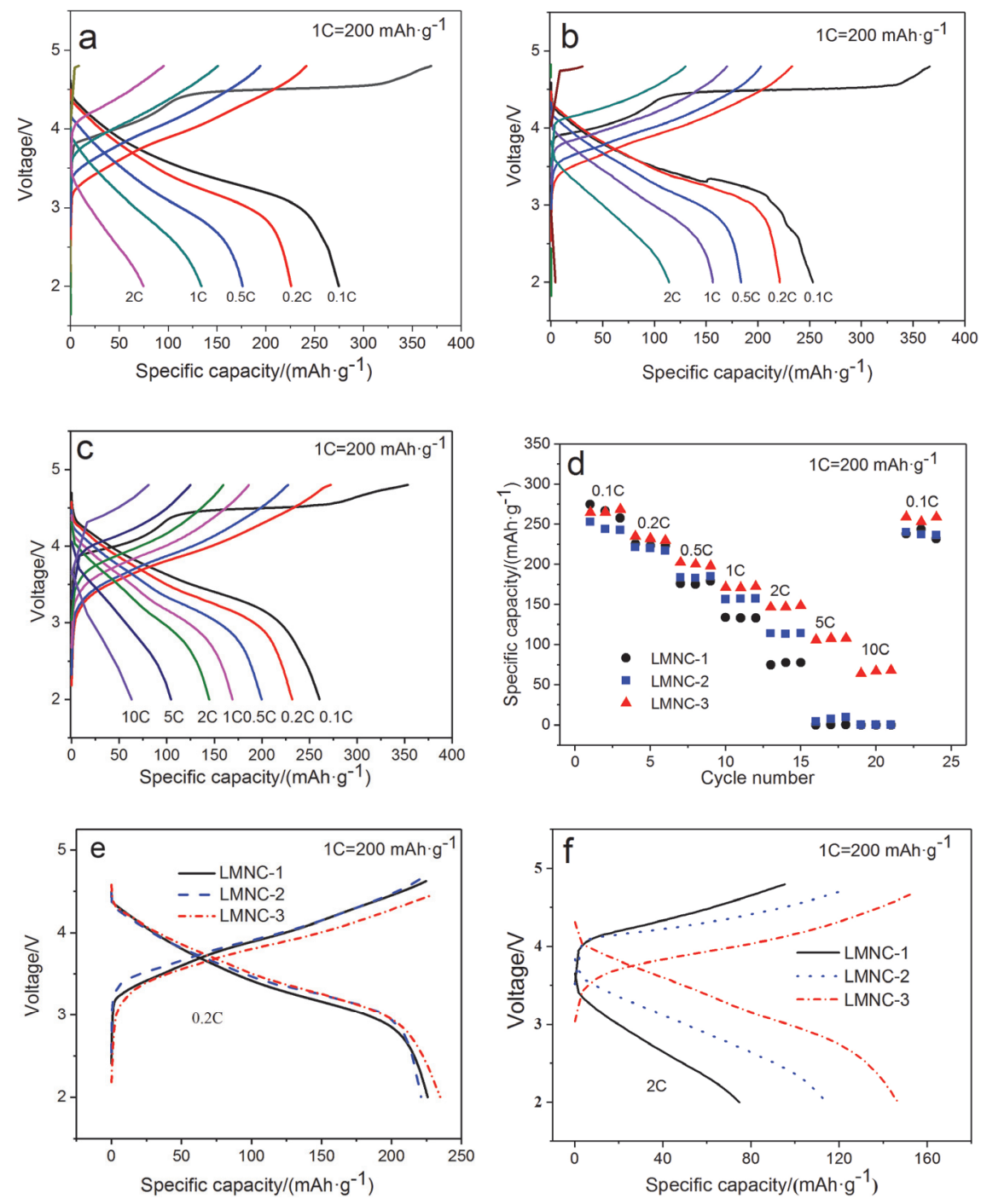

图 5 (a) LMNC-1, (b) LMNC-2 和(c) LMNC-3 不同倍率下的充放电曲线; (d) 三种材料倍率性能对比; (e, f) 三种材料在 $0.2 \mathrm{C}$ 和 $2 \mathrm{C}$ 下平台对比

Figure 5 Charge and discharge curves of LMNC-1 (a), LMNC-2 (b) and LMNC-3 (c) at different magnifications; (d) comparison of the performance of the three materials at rates; (e) and (f) platform comparison of the three materials at $0.2 \mathrm{C}$ and $2 \mathrm{C}$ 
$\mathrm{mAh} \cdot \mathrm{g}^{-1}$, 而只经过掺杂的 LMNC-2 容量保持率虽然高, 但是容量从 $167 \mathrm{mAh} \cdot \mathrm{g}^{-1}$ 降到 $137 \mathrm{mAh} \cdot \mathrm{g}^{-1}$, 这相对于 LMNC-3 容量要低. 为进一步说明双效改性对循环的作 用, 图 $5 \mathrm{~b}$ 对比了 LMNC-1、LMNC-2 和 LMNC-3 在 $0.5 \mathrm{C}$ 下循环 150 圈的平台衰减情况, 可以从图中发现在第一 圈是 LMNC-3 平台稍高, 在循环 150 圈后经过改性的 LMNC-3 有更高的平台, 而只经过 $\mathrm{Al}$ 掺杂的 LMNC-2 循环 150 圈后平台和 LMNC-1 的 150 圈的平台相差不大, 这说明 $\mathrm{Li}_{2} \mathrm{WO}_{4}$ 包覆减缓了富锂锰基材料的平台电压的 衰减. 众所周知, 富锂锰基材料放电平台电压衰减主要 由于材料表面从层状结构向尖晶石结构的转变, 产生相 变, 导致 $\mathrm{Li}^{+}$传输通道受阻, 进而产生电压衰减. 通过掺 杂稳定材料晶体结构, 材料表面包覆后抑制富锂材料尖 晶石转变, 减缓了放电电压平台的衰减 ${ }^{[15,25]}$.
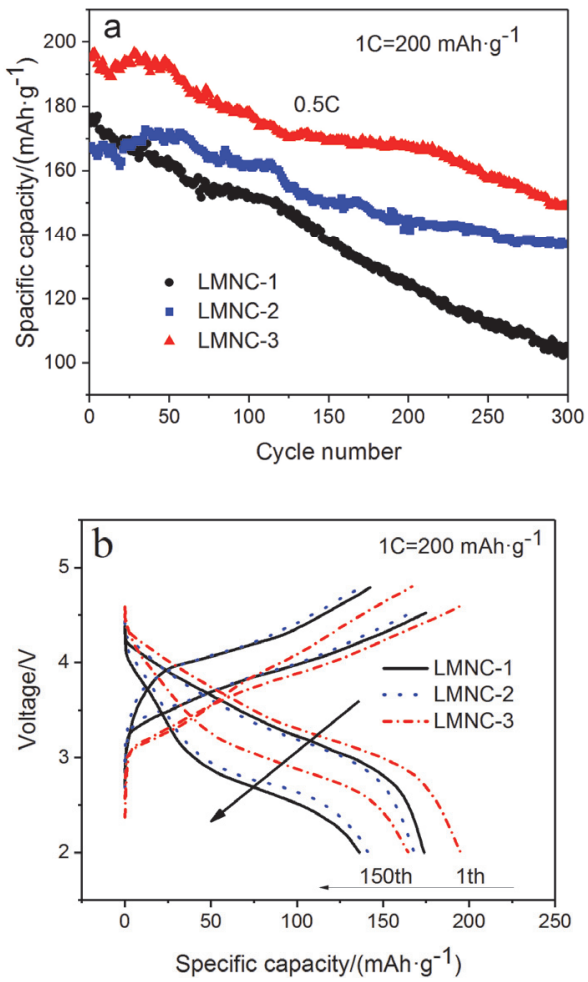

图 6 (a)三种材料在 $0.5 \mathrm{C}$ 电流密度下循环对比图; (b)平台对比

Figure 6 (a) cyclic comparison of three materials at a current density of $0.5 \mathrm{C}$; (b) platform comparison

至于 $\mathrm{Al}$ 掺杂改善富锂锰基循环稳定性能原因, 有 研究表明经过 $\mathrm{Al}$ 掺杂的富锂锰基材料, $\mathrm{Al}$ 替代了 $\mathrm{Mn}$ 的 位置稳定了材料的结构, 而且由于电荷转移效应抑制了 材料的电化学反应过程中尖晶石相的转移, 从而提高了 材料的循环性能 ${ }^{[33-34,42]}$. 包覆 $\mathrm{Li}_{2} \mathrm{WO}_{4}$ 后可以改善平台 衰减问题, 这是由于经过 $\mathrm{Li}_{2} \mathrm{WO}_{4}$ 包覆后提高了 $\mathrm{Li}^{+}$导电 性 ${ }^{[35-36]}$, 更有利 $\mathrm{Li}^{+}$脱嵌. 为了进一步证明其包覆的作 用, 图 7 是 $\mathrm{LMNC}-2$ 和 $\mathrm{LMNC}-3$ 在 $0.5 \mathrm{C}$ 电流密度下循 环第一圈和经过 150 圈循环的微分电容曲线对比图, 可 以从图中发现经过包覆 $\mathrm{Li}_{2} \mathrm{WO}_{4}$ 的 $\mathrm{LMNC}-3$ 放电平台明

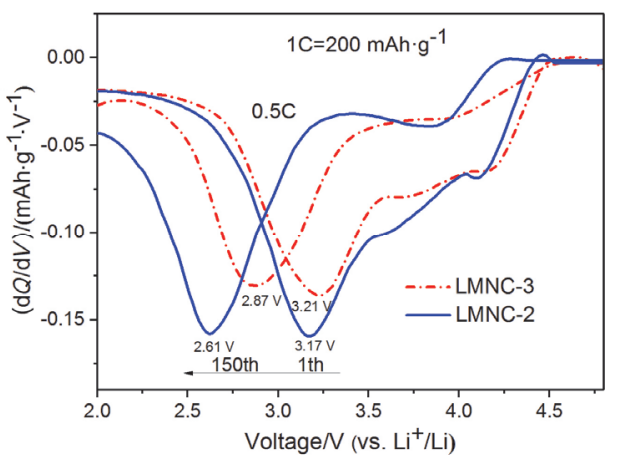

图 7 LMNC-2 和 LMNC-3 的 dQ/dV 曲线对比

Figure 7 Comparison of $\mathrm{dQ} / \mathrm{dV}$ curves between LMNC-2 and LMNC-3

显要高, 而且衰减相对也缓慢, 另外从图中也发现 LMNC- 2 电势差为 $0.56 \mathrm{~V}$ 左右, 而 $\mathrm{LMNC}-3$ 的电势差仅 有 $0.34 \mathrm{~V}$ 左右, 因此可以说明经过包覆的材料极化明显 减少，这都有利于改善材料的电化学性能.

图 8 是三种材料经过 300 圈循环后的电化学阻抗图 (EIS). 观察 Nyquist 图可以发现, 三种材料均是由高频 区的半圆部分和低频区的斜线部分组成. 拟合的等效电 路图由以下部分组成: 电解液和隔膜的欧姆阻抗 $R_{\mathrm{s}}$ 、电 荷转移阻抗 $R_{\mathrm{ct}}$ 、常相位角元件 CPE、Warburg 阻抗 $Z_{\mathrm{w}}$; 首 先 $R_{\mathrm{ct}}$ 与 $\mathrm{CPE}$ 并联, 再串联 $Z_{\mathrm{w}}$, 最后将 $R_{\mathrm{s}}$ 串联. 三种材 料的高频区半圆直径从小到大依次是 LMNC-3、 LMNC-2、LMNC-1, 说明经过改性的材料提高了材料的 电子导电率; 从低频区可以发现, LMNC-3 的斜率最大, 说明改性后的材料 $\mathrm{Li}^{+}$的扩散速率更大. 这是由于 $\mathrm{Al}$ 掺 杂不仅稳定材料结构, 而且材料表面重构减小了界面阻 抗 ${ }^{[46]}, \mathrm{Li}_{2} \mathrm{WO}_{4}$ 包覆后具有良好的 $\mathrm{Li}^{+}$导电性 ${ }^{[37-39]}$.

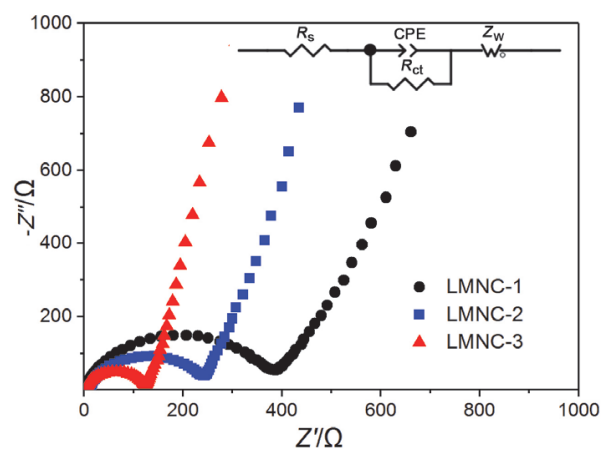

图 8 LMNC-1, LMNC-2 和 LMNC-3 材料循 300 圈后的 Nyquist 曲线 Figure 8 Nyquist curves of LMNC-1, LMNC-2 and LMNC-3 after 300 cycles

\section{3 结论}

采用溶胶-凝胶法成功实现了对富锂锰基进行 $\mathrm{Al}$ 掺 杂和 $\mathrm{Li}_{2} \mathrm{WO}_{4}$ 包覆的双效改性后，不仅提高了材料的倍 率性能，改善了长循环稳定性而且减缓了平台衰减问 题. 证明 $\mathrm{Al}$ 掺杂可以稳定材料的晶体结构, 从而改善富 锂锰基材料的倍率和循环性能; 包覆 $\mathrm{Li}_{2} \mathrm{WO}_{4}$ 后明显可 
以提高材料的倍率性能和减缓平台的衰减, 这是由于 $\mathrm{Li}_{2} \mathrm{WO}_{4}$ 具有良好的 $\mathrm{Li}^{+}$导电性, 并且包覆后还降低了电 池界面阻抗和保护材料不受电解液腐蚀, 减少了材料内 部极化. 该工作为今后富锂锰基改性研究提供了新的思 路和方向.

\section{4 实验部分}

\section{1 材料的合成}

按照富锂锰基 $\mathrm{Li}_{1.2} \mathrm{Mn}_{0.54-x} \mathrm{Al}_{x} \mathrm{Ni}_{0.13} \mathrm{Co}_{0.13} \mathrm{O}_{2}$ 正极材料 的化学计量比将 $\mathrm{CH}_{3} \mathrm{COOLi} \cdot 2 \mathrm{H}_{2} \mathrm{O} 、\left(\mathrm{CH}_{3} \mathrm{COO}\right)_{2} \mathrm{Mn}$ ・ $4 \mathrm{H}_{2} \mathrm{O} 、 \mathrm{Ni}\left(\mathrm{CH}_{3} \mathrm{COO}\right)_{2} \bullet 4 \mathrm{H}_{2} \mathrm{O} 、 \mathrm{Co}\left(\mathrm{CH}_{3} \mathrm{COO}\right)_{2} \bullet 4 \mathrm{H}_{2} \mathrm{O}$ 、 $\mathrm{Al}\left(\mathrm{NO}_{3}\right)_{3} \cdot 9 \mathrm{H}_{2} \mathrm{O}$ (其中 $\mathrm{CH}_{3} \mathrm{COOLi} \cdot 2 \mathrm{H}_{2} \mathrm{O}$ 过量 $5 \%$; 取 $x=0$ 和 0.03 )加水搅拌至完全溶解, 再加入适量的庶糖摚拌 配位 $1 \sim 2 \mathrm{~h}$; 再在 $85{ }^{\circ} \mathrm{C}$ 水浴中蒸干溶剂形成紫色透明 溶胶, 最后将溶胶置于 $100{ }^{\circ} \mathrm{C}$ 烘箱烘干. 将干凝胶研磨 成粉, 热处理分两步进行, 首先在空气中 $450{ }^{\circ} \mathrm{C}$ 敾烧 5 $\mathrm{h}$, 之后在空气中 $900{ }^{\circ} \mathrm{C}$ 炦烧 $12 \mathrm{~h}$ 得到相应的富锂正极 材料.

取制备好的 $\mathrm{Li}_{1.2} \mathrm{Mn}_{0.51} \mathrm{Al}_{0.05} \mathrm{Ni}_{0.13} \mathrm{Co}_{0.13} \mathrm{O}_{2}$, 加入配好 的 $\left(\mathrm{NH}_{4}\right)_{10} \mathrm{~W}_{12} \mathrm{O}_{41} \cdot x \mathrm{H}_{2} \mathrm{O}$ 和 $\mathrm{CH}_{3} \mathrm{COOLi} \cdot 2 \mathrm{H}_{2} \mathrm{O}$ 的混合溶液 中, 然后超声分散 $30 \mathrm{~min}$, 接着在负压下保持 5 到 $10 \mathrm{~h}$, 之后蒸干溶剂, 烘干压实后在 $650{ }^{\circ} \mathrm{C}$ 的空气中衤烧 $6 \mathrm{~h}$ 得到 5\% $\mathrm{Li}_{2} \mathrm{WO}_{4} @ \mathrm{Li}_{1.2} \mathrm{Mn}_{0.51} \mathrm{Al}_{0.03} \mathrm{Ni}_{0.13} \mathrm{Co}_{0.13} \mathrm{O}_{2}$.

\section{2 材料表征}

采用德国制造的 Bruker D8 Advance X 射线衍射仪 (XRD)分析样品的物相, 以 $\mathrm{Cu}$ 靶 $\mathrm{K} \alpha$ 为辐射源, $\lambda=$ $0.15406 \mathrm{~nm}$, 管电流为 $40 \mathrm{~mA}$, 管电压为 $40 \mathrm{kV}$, 扫描范 围是 $10^{\circ} \leqslant 2 \theta \leqslant 90^{\circ}$, 扫描速度为 $10.0\left(^{\circ}\right) / \mathrm{min}$, 步长是 $0.02^{\circ}$. 采用日本电子 JSM-4800 型场发射扫描电子显微 镜 FESEM 对合成的样品进行形貌分析, 其中工作电压 为 $5 \mathrm{kV}$, 工作电流为 $0.01 \mathrm{~mA}$.

\section{3 材料电化学性能测试}

将制备好的 LMNC-1、LMNC-2 和 LMNC-3 作为正 极活性物质, Super P-KS 6(质量比为 $1: 1$ )为导电剂, 聚 偏氟乙烯(PVDF)作为粘合剂, 按照 $7: 2: 1$ 的质量比加 入到适量的 $N$-甲基吡咯烷酮(NMP)的分散剂中混合摚 拌均匀制成浆料, 在集流体铝箔上进行涂布, 然后将涂 布后的极片在氩气气氛下 $100{ }^{\circ} \mathrm{C}$ 恒温干燥 $12 \mathrm{~h}$. 将干燥 后的极片作为正极, 金属锂片作为对电极, 聚丙烯微孔 膜(Celgard 2400)作为隔膜, 以 $1 \mathrm{~mol} \cdot \mathrm{L}^{-1} \mathrm{LiPF}_{6}$ 的碳酸甲 乙酯(DMC)-乙烯碳酸酯( $\mathrm{EC})$-碳酸甲乙酯 $\left(V_{\mathrm{DMC}}: V_{\mathrm{EC}}\right.$ : $\left.V_{\mathrm{EMC}}=1: 1: 1\right)$ 为电解液, 在充满氩气的干燥手套箱中 组装 CR2025 扣式半电池. 通过新威充放电测试仪在室 温下测试不同倍率下的充放电性能和长循环性能, 其中 充放电电压区间为 $2.0 \sim 4.8 \mathrm{~V}$, 电流密度 $1 \mathrm{C}=200 \mathrm{~mA}$ $\mathrm{g}^{-1}$. 通过普林斯顿电化学工作站进行材料的电化学阻 抗谱(EIS)测试.

\section{References}

[1] Tarascon, J.-M.; Armand, M. Nature 2001, 414, 359.

[2] Armand, M.; Tarascon, J.-M. Nature 2008, 451, 652.

[3] Park, K.-S.; Cho, M.-H.; Jin, S.-J.; Nahm, K.-S.; Hong, Y.-S. Solid State Ionics 2004, 171, 141.

[4] Kang, K.; Meng, Y.-S.; Breger, J.; Grey, C.-P.; Ceder, G. Science 2006, 311, 977.

[5] Fergus, J.-W. J. Power Sources 2010, 195, 939.

[6] Mohanty, D.; Kalnaus, S.; Meisner, R.-A.; Rhodes, K.-J.; Li, J.-L.; Payzant, E.-A.; Wood III, D.-L.; Daniel, C. J. Power Sources 2013, $229,239$.

[7] Gallagher, K.-G.; Croy, J.-R.; Balasubramanian, M.; Bettge, M.; Abraham, D.-P.; Burrell, A.-K.; Thackeray, M.-M. Electrochem. Commun. 2013, 33, 96.

[8] Croy, J.-R.; Gallagher, G.-K.; Balasubramanian, M.; Chen, Z.-H.; Ren, Y.; Kim, D.-H.; Kang, S.-H.; Dees, D.-W.; Thackeray, M.-M. J. Phys. Chem. C 2013, 117, 6525.

[9] Liu, S.; Liu, Z.-P.; Shen, X.; Wang, X.-L.; Liao, S.-C.; Yu, R.-C.; Wang, Z.-X.; Hu, Z.-W.; Chen, C.-T.; Yu, X.-Q.; Yang, X.-Q.; Chen, L.-Q. Adv. Eng. Mater. 2019, 9, 1901530.

[10] Zhang, J.-N.; Li, Q.-H.; Ouyang, C.-Y.; Yu, X.-Q.; Ge, M.-Y.; Huang, X.-J.; Hu, E.-Y.; Ma, C.; Li, S.-F.; Xiao, R.-J.; Yang, W.-L.; Chu, Y.; Liu, Y.-J.; Yu, H.-G.; Yang, X.-Q.; Huang, X.-J.; Chen, L.-Q.; Li, H. Natural Energy 2019, 4, 594.

[11] He, W.; Yuan, D.-D.; Qian, J.-F.; Ai, X.-P.; Yang, H.-X.; Cao, Y.-L. J. Mater. Chem. A 2013, 1, 11397.

[12] Li, Q.; Li, G.-S.; Fu, C.-C.; Luo, D.; Fan, J.-M.; Li, L.-P. ACS Appl. Mater. Interfaces 2014, 6, 10330 .

[13] Xiang, Y.-H.; Li, J.; Wu, X.-W.; Liu, Z.-X.; Xiong, L.-Z.; He, Z.-Q.; Yin, Z.-L. Ceram. Int. 2016, 42, 8833

[14] Knight, J.-C.; Nandakumar, P.; Kan, W.-H.; Manthiram, A. J. Mater. Chem. A 2015, 3, 2006.

[15] Deng, Z.-Q.; Manthiram, A. J. Phys. Chem. C 2011, 115, 7097.

[16] Du, J.-Y.; Shan, Z.-Q.; Zhu, K.-L.; Liu, X.-Y.; Tian, J.-H.; Du, H.-Y. J. Solid State Electrochem. 2014, 19, 1037.

[17] Yu, S.-H.; Yoon, T.; Mun, J.-Y.; Park, S.-J.; Kang, Y.-S.; Park, J.-H.; Oh, S.-M.; Sung, Y.-E. J. Mater. Chem. A 2013, 1, 2833.

[18] Li, L.; Song, B.-H.; Chang, Y.-L.; Xia, H.; Yang, J.-R.; Lee, K.-S.; Lu, L. J. Power Sources 2015, 283, 162.

[19] An, J.; Shi, L.-Y.; Chen, G.-R.; Li, M.; Liu, H.-J.; Yuan, S.; Chen, S.-M.; Zhang, D.-S. J. Mater. Chem. A 2017, 5, 19728.

[20] Li, Z.; Wang, Z.; Ban, L.-Q.; Wang, J.-T.; Lu, S.-G. Acta Chim Sinica 2019, 77, 1115. (李钊, 王忠, 班丽卿, 王建涛, 卢世刚, 化 学学报, 2019, 77, 1115.)

[21] Lim, S.-N.; Seo, J.-Y.; Jung, D.-S.; Park, S.-B.; Yeonc, S.-H. J. Electroanal. Chem. 2015, 740, 88.

[22] Hua, S.-L.; Cheng, G.-H.; Cheng, M.-Y.; Hwang, B.-J.; Santhanama, R. J. Power Sources 2009, 188, 554

[23] Zheng, J.-M.; Li, J.; Zhang, Z.-R.; Guo, X.-J.; Yang, Y. Solid State Ionics 2008, 179, 1794

[24] Zhang, X.-F.; Belharouak, I.; Li, L.; Lei, Y.; Elam, J.-W.; Nie, A.; Chen, X.-Q.; Yassar, R.-S.; Axelbaum, R.-L. Adv. Eng. Mater. 2013, 3, 1299.

[25] Wu, Q.; Yin, Y.-F.; Sun, S.-W.; Zhang, X.-P.; Wan, N.; Bai, Y. Electrochim. Acta 2015, 158, 73

[26] Wu, Y.; Muruga, V.-A.; Manthiram, A. J. Electrochem. Soc. 2008, 155, A635.

[27] Li, C.-D.; Yao, Z.-L.; Xu, J.; Tang, P.; Xiong, X. Ionics 2016, 23, 549.

[28] Ma, D.-T.; Zhang, P.-X.; Li, Y.-L.; Ren, X.-Z. Scientific Reports 2015, 5, 11257 .

[29] Xiang, Y-H.; Yin, Z.-L.; Zhang, Y.-H.; Li, X.-H. Electrochim. Acta 2013, 19, 214.

[30] Chen, Y.; Xu, G.-F.; Li, J.-L.; Zhang, Y.-K.; Chen, Z.; Kang, F.-Y. Electrochim. Acta 2013, 87, 686.

[31] Song, C.-K.; Feng, W.-J.; Su, W.-X.; Chen, L.-J.; Li, M.-M. Integrated Ferroelectrics 2019, $200,117$.

[32] Huang, X.-K.; Zhang, Q.-S.; Chang, H.-T.; Gan, J.-L.; Yue, H.-J.; Yang, Y. J. Electrochem. Soc. 2009, 156, A162.

[33] Li, Z.; Chernova, N.-A.; Feng, J.-J.; Upreti, S.; Omenya, F.; Whittingham, M.-S. J. Electrochem. Soc. 2012, 159, A116.

[34] Yan, W. C.; Xie, Y.; Jiang, J.-C.; Sun, D.-Y.; Ma, X.-D.; Lan, Z.-G.; Jin, Y.-C. ACS Sustainable Chem. Eng. 2018, 6, 4625.

[35] Yahaya, A.-H.; Ibrahim, Z.-A.; Arof, A.-K. J. Alloys Compd. 1996, 
241,147

[36] Nassau, K. J. Electrochem. Soc. 1980, 127, 2743

[37] Hayashi, T.; Okada, J.; Toda, E.; Kuzuo, R.; Matsuda, Y.; Kuwata, N.; Kawamura, J. J. Power Sources 2015, 127, 2743.

[38] Hayashi, T.; Matsuda, Y.; Kuwata, N.; Kawamura, J. J. Power Sources 2017, 354, 41.

[39] Hayashi, T.; Miyazaki, T.; Matsuda, Y.; Kuwata, N.; Saruwatari, M.; Furuichi, Y.; Kurihara, K.; Kuzuo, R.; Kawamura J. J. Power Sources 2016, 305, 46.

[40] Li, X.-N.; Cao, Z.-X.; Dong, H.-Y.; Shi, Z.-P.; Zhang, H.-S.; Li, J.-Y.; Yang, S.-J.; Yang, S.-T. RSC Adv. 2020, 10, 3166.

[41] Yue, P.; Wang, Z.-X.; Guo, H.-J.; Xiong, X.-H.; Li, X.-H. Electrochim. Acta 2013, 92, 1 .
[42] Nayak, P.-K.; Grinblat, J.; Levi, M.; Levi, E.; Kim, S.; Choi, W.-J.; Aurbach, D. Adv. Eng. Mater. 2016, 6, 1502398.

[43] Thackeray, M.-M.; Kang, S.-H.; Johnson, C.-S.; Vaughey, J.-T.; Benedeka, R.; Hackneyb, S.-A. J. Mater. Chem. 2007, 115, 305.

[44] Zheng, Z.-H.; Li, Q. Introduction to Rietveld Refinement with X-Ray Power Diffraction Data GSAS Software, China Building Materials Press, Beijing, 2016. (郑振环, 李强, $X$ 射线多晶衍射数据 Rietveld 精修及 GSAS 软件入门, 中国建材工业出版社, 北京, 2016.)

[45] Guilmard, M.; Rougier, A.; Grüne, M.; Croguennec, L.; Delmas, C. J. Power Sources 2003, 115, 305.

[46] Guo, H.-C.; Xia, Y.-G.; Zhao, H.; Yin, C.; Ji, K.; Zhao, F.; Liu, Z.-P. Ceramics International 2017, 43, 13845.

(Cheng, B.) 